\title{
A Multiexposure Technology for Sampled Bragg Gratings and its Applications in Dual-Wavelength Lasing Generation and OCDMA En/Decoding
}

\author{
Jingsi Li, Student Member, IEEE, Yun Cheng, Zuowei Yin, Linghui Jia, Xiangfei Chen, Member, IEEE, \\ Shengchun Liu, Simin Li, and Yanqing Lu, Senior Member, IEEE
}

\begin{abstract}
In this letter, a multiexposure technology for sampled Bragg gratings is proposed and investigated for the first time. Combined with reconstruction-equivalent-chirp technology, the useless zeroth-order channel can be eliminated and the \pm 1 st-order channels can be enhanced significantly. The proposed method is applied experimentally to achieve a dual-wavelength fiber laser and is studied both numerically and experimentally in two ways for the optical code-division multiple access en/decoding system. Its properties may lead to more efficient design and fabrication of grating-based optical devices.
\end{abstract}

Index Terms-Bragg gratings, dual-wavelength fiber laser, optical code-division multiple access (OCDMA) en/decoding.

\section{INTRODUCTION}

B RAGG gratings have proven attractive in a wide variety of fiber-optic applications [1]-[4], such as distributed-feedback (DFB) lasers, optical code-division multiple access (OCDMA) en/decoders, dispersion-compensating gratings, fiber sensors, etc. To realize complex filter functions, complicated phase shifts and chirps are needed, which require expensive and precise nanometer-scale fabrication. To overcome this limit, reconstruction-equivalent-chirp (REC) technology has been proposed to produce desired equivalent phase shifts and chirps in all nonzero-order channels of a sampled Bragg grating (SBG) [5] by modulating sampling periods under a submicrometer precision. However, although many fiber-optic components have been realized by REC technology (e.g., recording OCDMA en/decoders [6], DFB fiber lasers [7], semiconductor lasers [8] and special filters [9]), the basic sampling structure makes at least half of the grating useless, which greatly decreases the efficiency of the devices. Usually the \pm 1 st-order channels have the biggest effective index modulation among all nonzero-order channels. However, even if the duty cycle is fixed as 0.5 to reach the maximum effective index modulation, the \pm 1 st-order channels are born weak at about $1 / 3$ of the peak index modulation (index modulation in uniform Bragg gratings) and at about $2 / 3$ of the zeroth-order channel

Manuscript received March 21, 2009; revised July 15, 2009. First published August 25, 2009; current version published October 16, 2009. This work was supported by the National "863" Project of China (2007AA03Z417), by the National Natural Science Foundation of China under Grant 60877043, and by the New-Century Excellent Talents Supporting Program of the Ministry of Education, China.

The authors are with the National Laboratory of Microstructures, Nanjing University, Nanjing 210093, China (e-mail: lijingsi@gmail.com).

Color versions of one or more of the figures in this letter are available online at http://ieeexplore.ieee.org.

Digital Object Identifier 10.1109/LPT.2009.2030877

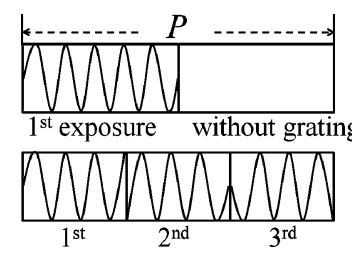

(a)

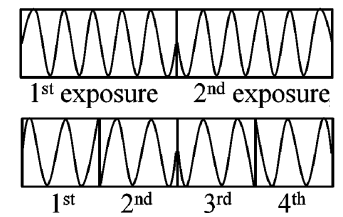

Fig. 1. (a) Illustration of a single sampling period in OSBG; (b) DESBG (c) TESBG; (d) QESBG. There are three general steps to fabricate a DESBG. 1) The hydrogen-loaded fiber is illuminated by a 244-nm ultraviolet (UV) laser beam through a phasemask. An OSBG is formed through turning ON and OFF the laser beam moved by a computer-controlled submicrometer precision transducer (first exposure). 2) Move the UV laser beam back to the original position and then move the phasemask by a PZT to obtain the desired phase difference. 3) Write the grating in the second part of the sampling period in the same way as step 1 (second exposure). To fabricate a TESBG or a QESBG, steps 2 and 3 need to be repeated two or three times.

of SBG. In this letter, a multiexposure technology is proposed. In a double-exposure SBG (DESBG) with antiphase gratings, the zeroth and all even-order channels are eliminated and the strengths of all odd-order channels are doubled. Triple-exposure SBG (TESBG) and quadruple-exposure SBG (QESBG) are studied numerically to indicate that it is possible to eliminate one of the \pm 1 st-order channels and to further enhance the other one. The combination of the multiexposure technology and the REC technology may lead to better performance of low-cost and high-end grating components.

\section{PRINCIPLE}

Equivalent phase shifts and chirps can be achieved by adjusting the sampling periods of an SBG [7]-[9]. Commonly, a period of an original SBG (OSBG) is divided into two equal parts (i.e., with a duty cycle of 0.5 ), and the grating is written only in the first part. In multiexposure technology, every sampling period $P$ is divided into $n$ equal parts, and the gratings with the same grating pitch are written in each part with different phases. Fig. 1 gives an illustration of a single period of OSBG, DESBG, TESBG, and QESBG. According to Fourier analysis, the Fourier coefficient of the $m$ th-order channel can be

$$
F_{m}=\frac{1}{P} \int_{0}^{p} s(z) \cdot e^{-i m \pi z /(P / 2)} \cdot d z
$$

where $z$ is the position along the fiber and $s(z)$ is the unit sampling function. In an OSBG or a DESBG, the Fourier coefficients correspond to the grating strength, and for the zeroth and 


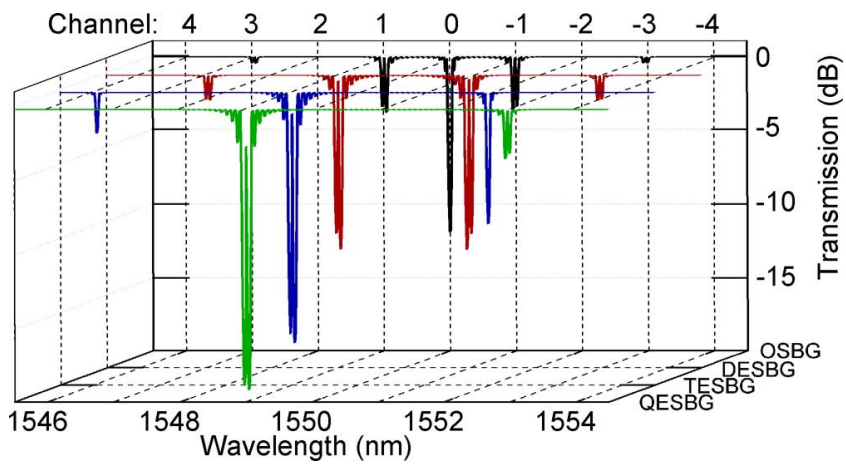

Fig. 2. Calculated transmission spectra of an OSBG, a DESBG, a TESBG, and a QESBG with $\pi$ equivalent phase shifts provided by REC technology.

\pm 1 st channels, they can be obtained by

$$
F_{0}=\frac{1}{2}(A+B) \quad F_{ \pm 1}=\frac{1}{i \pi}(A-B) .
$$

$A$ and $B$ correspond to $s(z)$ of the first and the second part of a sampling period. For an OSBG, grating is imprinted only in the first part of the sampling period $(A=1$ and $B=0)$, then $\left|F_{0}\right|=0.5$ and $\left|F_{ \pm 1}\right|=0.32$. For a DESBG, when an antiphase grating is written on the second part $(A=1$ and $B=e^{i \pi}=-1$ ), then $\left|F_{0}\right|=0$ and $\left|F_{ \pm 1}\right|=0.64$, which means the zeroth-order channel is eliminated and the strength of the \pm 1 st-order channels is doubled.

As for TESBG, the Fourier coefficients can be obtained by

$$
\begin{aligned}
F_{0} & =\frac{(A+B+C)}{3} \\
F_{ \pm 1} & =\frac{\left(e^{\mp i 2 \pi / 3}-1\right)}{i 2 \pi}\left(A+B e^{\mp i 2 \pi / 3}+C e^{\mp i 4 \pi / 3}\right) \\
F_{ \pm 2} & =\frac{\sqrt{3}}{2 \pi}\left(A+B e^{\mp i 4 \pi / 3}+C e^{\mp i 8 \pi / 3}\right) \quad F_{ \pm 3}=0
\end{aligned}
$$

where $C$ corresponds to $s(z)$ in the third part. It can be seen that the \pm 1 st-order channels are no longer equal. When the phases of the three parts are $0,2 \pi / 3$ and $4 \pi / 3$, the Fourier coefficients can be obtained as: $\left|F_{0}\right|=0,\left|F_{1}\right|=0.83,\left|F_{-1}\right|=0,\left|F_{2}\right|=$ 0 , and $\left|F_{-2}\right|=0.41$. Only the 1 st and -2 nd channels exist from the -3 rd to the third-order channels. More importantly, the effective index modulation of the first channel reaches $83 \%$ of the peak index modulation.

Similarly, in a QESBG, when the phases of the four parts are $0, \pi / 2, \pi$, and $3 \pi / 2$, only the 1 st and -3 rd channels exist from the -4 th to the 4 th channels, with $\left|F_{1}\right|=0.90$ and $\left|F_{-3}\right|=$ 0.30 .

Conclusively, in an $n$-time exposure SBG, when the phases of the $m$ th part is $m \times 2 \pi / n$, the first-order channel can reach $n / \pi \times \sin (\pi / n) \times 100 \%$ of the uniform Bragg grating strength, and the second biggest channel is $(1-n)$ th, with

$$
F_{1-n}=\frac{n}{-i 2(1-n) \pi}\left[e^{-i 2(1-n) \pi / n}-1\right] .
$$

Fig. 2 shows the calculated transmission spectra of an OSBG, a DESBG, a TESBG, and a QESBG for comparison, which clearly shows the enhancement of the first-channel and the weakening of other channels.

Fig. 3 shows an experimental transmission spectrum of a DESBG with $\pi$ equivalent phase shifts. The zeroth-order

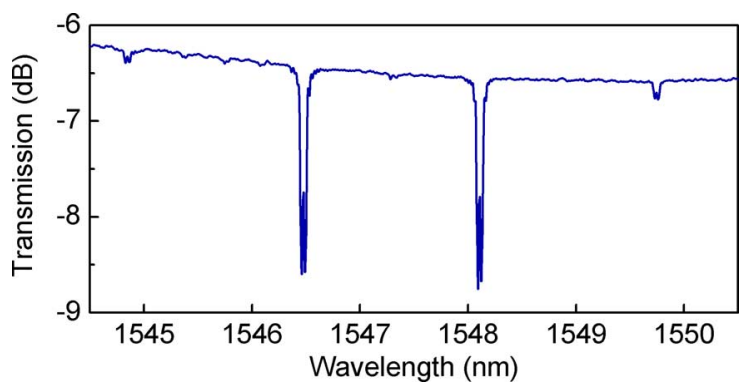

Fig. 3. Transmission spectrum of an experimental realized DESBG with $\pi$ equivalent phase shifts provided by REC technology.

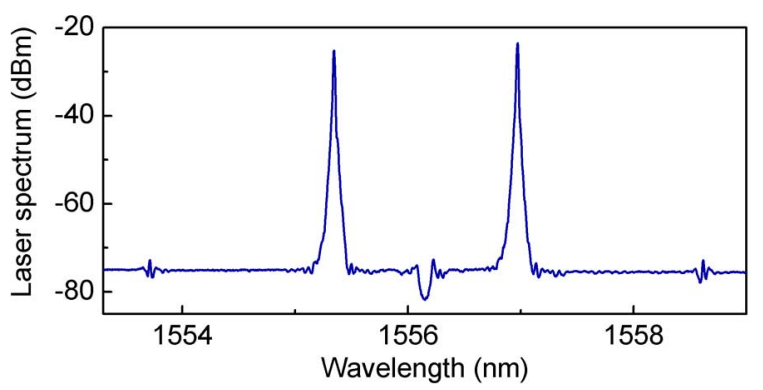

Fig. 4. Experimental realized dual-wavelength fiber laser based on multiexposure technology and REC technology. Two lasing wavelengths are at 1555.34 and $1556.97 \mathrm{~nm}$.

channel is eliminated as desired, and four equivalent phase shifts are located separately in the \pm 1 st and \pm 3 rd channels.

\section{APPLICATIONS}

\section{A. Dual-Wavelength Lasing Generation}

Dual-wavelength fiber lasers have been proposed and realized in many ways for potential uses such as fiber-optic sensing, optical instrument testing and microwave generating, etc. RECbased dual-wavelength lasers typically have some weaknesses. First, the two lasers are located in the -1 st-order channel, and the zeroth-order channel is always a major obstacle in that it may lase before the desired wavelengths [10]. Second, the two wavelengths cannot be easily and separately adjusted, which seriously affect the practical use of the devices. DESBG provides a way to fundamentally inhibit the zeroth-order channel, and the wavelength spacing between the two lasers can be controlled and adjusted easily in a wide range by altering the sampling period. The wavelength difference between the \pm 1 st-order channels is inversely proportional to the sampling period. As shown in Fig. 4, through eliminating or weakening the zeroth-order channel, a dual-wavelength laser is achieved with separate $\pi$ equivalent phase shifts based on a DESBG.

\section{B. OCDMA En/Decoding}

In an OCDMA system, $P / C$ is always treated as an important factor in evaluating performance, which is characterized by the ratio of the auto-correlation peak to the maximum cross-correlation [11]. A higher $P / C$ always means a better device property. Here the multiexposure technology along with REC technology is applied both numerically and experimentally to OCDMA en/decoders with different gold code sequences to examine the device performance change. The DESBG is used in two en/decoding ways and compared with en/decoders 
TABLE I

COMPARISON OF THE $P / C s$ IN THREE DifFERENT EN/DECODING METHODS

\begin{tabular}{|c|c|c|c|}
\hline \hline Value & $\begin{array}{c}\text { I: Using the } \\
-1^{\text {st }} \text { Channel } \\
\text { of an OSBG }\end{array}$ & $\begin{array}{c}\text { II: Using the } \\
-1^{\text {st }} \text { Channel } \\
\text { of a DESBG }\end{array}$ & $\begin{array}{c}\text { III:Using both } \\
\pm 1^{\text {st }} \text { Channels } \\
\text { of a DESBG }\end{array}$ \\
\hline Category & $0.41 \mathrm{~mm}$ & $0.41 \mathrm{~mm}$ & $0.82 \mathrm{~mm}$ \\
\hline Duty cycle & 0.5 & 0.5 & 0.5 \\
\hline Encoding bandwidth & $4 \mathrm{~nm}$ & $4 \mathrm{~nm}$ & $4 \mathrm{~nm}$ \\
\hline FWHM $^{\text {a }}$ & $2 \mathrm{ps}$ & $2 \mathrm{ps}$ & $2 \mathrm{ps}$ \\
\hline$\Delta \mathrm{n}^{\mathrm{b}}$ of 63 -chip & $1.5 \mathrm{e}^{-4}$ & $9 \mathrm{e}^{-5}$ & $5 \mathrm{e}^{-5}$ \\
\hline$\Delta \mathrm{n}$ of $127-$ chip & $1.5 \mathrm{e}^{-4}$ & $7.5 \mathrm{e}^{-5}$ & $3 \mathrm{e}^{-5}$ \\
\hline$P / C$ of $63-$ chip $\left(\mathrm{S}^{\mathrm{c}}\right)$ & $\mathbf{1 3 . 0 1 8}$ & $\mathbf{1 6 . 7 6 3}$ & $\mathbf{1 6 . 4 6 0}$ \\
\hline$P / C$ of $127-\operatorname{chip}(\mathrm{S})$ & $\mathbf{3 0 . 6 1 1}$ & $\mathbf{3 2 . 5 0 4}$ & $\mathbf{3 1 . 7 2 0}$ \\
\hline$P / C$ of $63-\operatorname{chip}\left(\mathrm{E}^{\mathrm{d}}\right)$ & $\mathbf{1 2 . 9 1 3}$ & $\mathbf{1 6 . 1 0 6}$ & $\mathbf{1 5 . 9 5 9}$ \\
\hline \hline
\end{tabular}

${ }^{\mathrm{a}} \mathrm{FWHM}$ : the full width at half maximum of the Gaussian pulse.

${ }^{\mathrm{b}} \Delta \mathrm{n}$ : the maximum index modulation.

${ }^{\mathrm{c}} \mathrm{S}$ : the $P / C$ is an average of $\sim 25$ results based on simulations.

${ }^{\mathrm{d}} \mathrm{E}$ : the $P / C$ is an average of $\sim 8$ results based on experiments.

based on OSBG (Type I) under the same code sequences and reflection level $(\sim 5 \mathrm{~dB})$. The first way (Type II) is the same as for an OSBG in that it uses the -1 st channel. And the second way (Type III) uses both \pm 1 st channels. The simulation of 63and 127-chip and experimental results of 63-chip are listed in Table I, which clearly indicates an improvement when using multiexposure technology. It is well known that superstructured fiber Bragg grating (SSFBG) has been successfully used in the OCDMA system [6], [11]. From [11] and Table I, 127-chip SSFBG and DESBG have almost the same $P / C$ performance.

Without the disturbance of the zeroth-order channel in an OSBG, Type II shows a better performance in both 63- and 127-chip en/decoding than Type I. Although Type III is not as good as Type II, it is still better than Type I. Since the power peak of the pulse in Type III is located at the center of the zeroth-order channel, the pulse energy is somewhat wasted, leading to a lower $P / C$ than Type II.

\section{DISCUSSION}

In the multiexposure technology, a piezoelectric transducer (PZT) is employed, which can be used directly in producing true phase-shifts or chirps. In such a situation, it is interesting to examine why the equivalent phase control is used instead of the true phase control. First, based on the REC technology, the equivalent phase-shifts and chirps are produced by changing the sampling period $P$, which is usually in the order of millimeter. The errors produced by the PZT only change the effective strength of all channels and have nothing to do with the phase shifts or chirps. For example, normally when an error of $50 \mathrm{~nm}$ occurs while using a PZT, the true phase shift will shift $\pm 0.2 \pi$, but when using multiexposure technology this only brings in a small zeroth-order channel, and the phase shift remains exactly $\pi$. Second, when more phase shifts and chirps are needed to achieve high-end gratings with complicated filtering functions, the use of a PZT multiple times will produce useless phase shifts and chirps due to PZT errors, but when relying on
REC technology in combination with the multiexposure method the number of phase shifts and chirps remains exactly the same, no matter how complex the structure is.

\section{CONCLUSION}

The REC technology has displayed good ability in producing high-performance and low-cost optical devices. However, the sampled gratings, on which the REC technology is based, have two main weaknesses: 1) when only one channel is used, the others, especially the zeroth-order channel, can become disturbances and might seriously affect the device property [7];2) the effective grating strength is much lower than that of the uniform grating, which results directly in a relative low performance. The proposed multiexposure technology will achieve high efficiency in solving these two problems. Using DESBG, efficiency can be doubled and a dual-wavelength fiber laser can be more easily achieved. The DESBG can also improve the performance of an OCDMA system. In a QESBG, the efficiency reaches $90 \%$ of that of the uniform grating. Furthermore, the effective strength and the phase shift can also be controlled simultaneously in an individual channel. Therefore, besides the advantages mentioned in this letter, we believe the multiexposure technology will make REC technology more flexible in fabricating high-end devices for high-capacity fiber-optic system which needs further exploration in the future.

\section{REFERENCES}

[1] A. Frank, K. Bohnert, K. Haroud, H. Brandle, C. V. Poulsen, J. E. Pedersen, and J. Patscheider, "Distributed feedback fiber laser sensor for hydrostaticpressure," IEEE Photon. Technol. Lett., vol. 15, no. 12, pp. 1758-1760, Dec. 2003.

[2] J. Hubner, P. Varming, and M. Kristensen, "Five wavelength DFB fiber laser source for WDM systems," Electron. Lett., vol. 33, no. 2, pp. 139-140, 1997.

[3] P. Teh, P. Petropoulos, M. Ibsen, and D. J. Richardson, "Phase encoding and decoding of short pulses at $10 \mathrm{~Gb} / \mathrm{s}$ using superstructured fiber Bragg gratings," IEEE Photon. Technol. Lett., vol. 13, no. 2, pp. 154-156, Feb. 2001.

[4] M. Ibsen, M. K. Durkin, M. J. Cole, and R. I. Laming, "Sinc-sampled fiber Bragg gratings for identical multiple wavelength operation," IEEE Photon. Technol. Lett., vol. 10, no. 6, pp. 842-844, Jun. 1998.

[5] B. J. Eggleton, P. A. Krug, L. Poladian, and F. Ouellette, "Long periodic superstructure Bragg gratings in optical fibres," Electron. Lett., vol. 30, no. 19, pp. 1620-1622, 1997.

[6] Y. Dai, X. Chen, Y. Zhang, J. Sun, and S. Xie, "Phase-error-free 1023 chip OCDMA en/de-coders based on reconstruction-equivalent-chirp technology and error-correction method," in Proc. OFC 2007, Paper JWA28.

[7] D. Jiang, X. Chen, Y. Dai, H. Liu, and S. Xie, "A novel distributed feedback fiber laser based on equivalent phase shift," IEEE Photon. Technol. Lett., vol. 16, no. 12, pp. 2598-2600, Dec. 2004.

[8] J. Li, H. Wang, X. Chen, Z. Yin, Y. Shi, Y. Lu, Y. Dai, and H. Zhu, "Experimental demonstration of distributed feedback semiconductor lasers based on reconstruction-equivalent-chirp technology," Opt. Express, vol. 17, pp. 5240-5245, 2009.

[9] Y. Dai, X. Chen, L. Xia, Y. Zhang, and S. Xie, "Sampled Bragg grating with desired response in one channel by use of a reconstruction algorithm and equivalent chirp," Opt. Lett., vol. 29, pp. 1333-1335, 2004.

[10] J. Sun, Y. Dai, Y. Zhang, X. Chen, and S. Xie, "Dual-wavelength DFB fiber laser based on unequalized phase shifts," IEEE Photon. Technol. Lett., vol. 18, no. 23, pp. 2493-2495, Dec. 1, 2006.

[11] X. Wang, K. Matsushima, A. Nishiki, N. Wada, and K. Kitayama, "High reflectivity superstructured FBG for coherent optical code generation and recognition," Opt. Express, vol. 12, pp. 5457-5468, 2004. 